\title{
Ly $\alpha$ Observations of High Radial Velocity Low-mass Stars Ross 1044 and Ross 825
}

\author{
Adam C. Schneider ${ }^{1}$ (D), Evgenya L. Shkolnik ${ }^{1}$ (D), Travis S. Barman ${ }^{2}$ (D), and R. Parke Loyd ${ }^{1}$ (D) \\ ${ }^{1}$ School of Earth and Space Exploration, Arizona State University, Tempe, AZ 85282, USA; aschneid10@ gmail.com \\ ${ }^{2}$ Lunar \& Planetary Laboratory, University of Arizona, 1629 E. University Blvd, Tucson, AZ 85721, USA \\ Received 2019 September 6; revised 2019 September 23; accepted 2019 September 26; published 2019 November 14
}

\begin{abstract}
The discovery of habitable zone (HZ) planets around low-mass stars has highlighted the need for a comprehensive understanding of the radiation environments in which such planets reside. Of particular importance is knowledge of the far-ultraviolet (FUV) radiation, as low-mass stars are typically much more active than solar-type stars and the proximity of their HZs can be one-tenth the distance. The vast majority of the flux emitted by low-mass stars at FUV wavelengths occurs in the Ly $\alpha$ line at $1216 \AA$. However, measuring a low-mass star's Ly $\alpha$ emission directly is almost always impossible because of the contaminating effects of interstellar hydrogen and geocoronal airglow. We observed Ross 825 (K3) and Ross 1044 (M0), two stars with exceptional radial velocities, with the Space Telescope Imaging Spectrograph on board the Hubble Space Telescope. Their radial velocities resulted in significant line shifts, allowing for a more complete view of their Ly $\alpha$ line profiles. We provide an updated relation between effective temperature and Ly $\alpha$ flux using Gaia DR2 astrometry as well as updated, model-independent relationships between Ly $\alpha$ flux and UV flux measurements from the Galaxy Evolution Explorer (GALEX) for lowmass stars. These new relations, in combination with GALEX's considerable spatial coverage, provide substantial predictive power for the $\mathrm{Ly} \alpha$ environments for thousands of nearby, low-mass stars.
\end{abstract}

Unified Astronomy Thesaurus concepts: Low mass stars (2050)

Supporting material: data behind figure

\section{Introduction}

Both K- and M-type stars have distinct advantages for studying exoplanets. $\mathrm{M}$ dwarfs are the most abundant stars in the Galaxy (Bochanski et al. 2010), have large planet occurrence rates (Dressing \& Charbonneau 2015; HardegreeUllman et al. 2019; Tuomi et al. 2019), have numerous observational advantages for exoplanet characterization, and have therefore emerged as targets for current and future exoplanet investigations. $\mathrm{K}$ dwarfs, while not as numerous as M dwarfs, also have substantial planet occurrence rates (Mulders et al. 2015), and the reduced X-ray and UV radiation incident on habitable zone (HZ) planet atmospheres around K dwarfs has led some to suggest that $\mathrm{K}$ dwarfs might be the most promising targets for the detection of biology outside of our solar system (Heller \& Armstrong 2014; Cuntz \& Guinan 2016; Arney 2019; Richey-Yowell et al. 2019). For these reasons, understanding the properties of both $\mathrm{K}$ and $\mathrm{M}$ dwarfs is an essential component of current and upcoming planethunting missions such as the Transiting Exoplanet Survey Satellite (TESS) and PLAnetary Transits and Oscillations of stars (PLATO; Rauer et al. 2014; Ricker et al. 2015).

The UV radiation emitted by low-mass stars can chemically modify, ionize, and even erode a planetary atmosphere over time, drastically affecting their habitability (Kasting et al. 1993; Lichtenegger et al. 2010; Segura et al. 2010; Hu et al. 2012; Luger \& Barnes 2015). Furthermore, several studies have shown that incident UV flux could lead to the formation of abiotic oxygen and ozone, resulting in potential false-positive biosignatures (Domagal-Goldman et al. 2014; Tian et al. 2014; Harman et al. 2015). The most prominent far-ultraviolet (FUV) emission line, Ly $\alpha(\lambda 1215.67 \AA)$, comprises $\sim 37 \%-75 \%$ of the total 1150-3100 $\AA$ flux from most late-type stars (France et al. 2013). Because $\operatorname{Ly} \alpha$ can affect the photodissociation of important molecules such as $\mathrm{H}_{2} \mathrm{O}$ and $\mathrm{CH}_{4}$, any photochemical models assessing potential biosignatures or atmospheric abundances will require accurate Ly $\alpha$ host star flux estimates (e.g., Rugheimer et al. 2015).

In almost all cases, Ly $\alpha$ cannot be directly observed because of (1) attenuation of photons by optically thick hydrogen absorption located in the intervening interstellar medium (ISM) and (2) contamination from Earth's own $\operatorname{Ly} \alpha$ geocoronal airglow. In fact, there exists only one complete Ly $\alpha$ spectrum in the Hubble Space Telescope (HST) archive, that of Kapteyn's star (an M1 subdwarf; Guinan et al. 2016). With a radial velocity (RV) of $245 \mathrm{~km} \mathrm{~s}^{-1}$, its stellar Ly $\alpha$ emission feature is shifted out of the geocoronal line core and ISM absorption.

For all other stars, Ly $\alpha$ emission may only be estimated. Wood et al. (2005) reconstructed Ly $\alpha$ fluxes by determining interstellar hydrogen column densities and velocities inferred from deuterium and metal lines. Alternatively, reconstructions have been performed by fitting 1 or 2 Gaussians to what remains of the wings of the Ly $\alpha$ profile (France et al. 2012; Youngblood et al. 2016, 2017). Such reconstructions have been used extensively to produce correlations between $\operatorname{Ly} \alpha$ fluxes and other spectral emission lines (Linsky et al. 2013) and broadband UV photometry (Shkolnik et al. 2014) from the Galaxy Evolution Explorer (GALEX). These correlations have been used to evaluate the capabilities of different spectral types to develop and sustain life (e.g., Cuntz \& Guinan 2016).

The lack of observational Ly $\alpha$ constraints on low-mass stars has limited progress in predicting the environments that surround them. In an effort to further inform models of lowmass stellar UV flux levels, we have extended the observational sample of stars for which the shape and majority of Ly $\alpha$ flux can be measured beyond just Kapteyn's star. We identified two low-mass targets, Ross 825 (K3) and Ross 1044 (M2). 
Table 1

Target Sample

\begin{tabular}{|c|c|c|c|c|}
\hline Property & Ross 825 & References & Ross 1044 & References \\
\hline 2MASS Name & $21111696+3331272$ & 1 & $15032457+0346574$ & 1 \\
\hline Spectral Type & K3 & 2 & M0 & 3 \\
\hline$\pi$ (mas) & $10.17 \pm 0.08$ & 4 & $26.9 \pm 8.4$ & 5 \\
\hline$\mu_{\delta}\left(\operatorname{mas} \mathrm{yr}^{-1}\right)$ & $159.31 \pm 0.29$ & 4 & $695.3 \pm 4.6$ & 5 \\
\hline $\mathrm{RV}\left(\mathrm{km} \mathrm{s}^{-1}\right)$ & $-340.17 \pm 0.67$ & 4 & $-169.55 \pm 1.80$ & 4 \\
\hline Age (Gyr) & $>10$ & 3 & $>10$ & 3 \\
\hline
\end{tabular}

References. (1) 2MASS, Skrutskie et al. (2006); (2) Bidelman (1985); (3) This work; (4) Gaia Collaboration et al. (2016, 2018); (5) Finch \& Zacharias (2016).

\section{Target Sample}

Targets for this program were chosen based on their spectral types (later than K0), distances (within $100 \mathrm{pc}$ ), and large absolute RVs. We chose a radial velocity threshold of $150 \mathrm{~km} \mathrm{~s}^{-1}$, which results in $\mathrm{a} \approx 0.6 \AA$ wavelength shift of the Ly $\alpha$ emission line. Such stars are rare because the vast majority of low-mass stars in the solar neighborhood have absolute radial velocities less than $50 \mathrm{~km} \mathrm{~s}^{-1}$. Shkolnik et al. (2012) found an average RV of $1.6 \mathrm{~km} \mathrm{~s}^{-1}$ with a standard deviation of $23.9 \mathrm{~km} \mathrm{~s}^{-1}$ for a sample of $159 \mathrm{M}$ dwarfs within $25 \mathrm{pc}$.

We identified two targets, Ross 825 and Ross 1044, that satisfied these criteria and were bright enough to acquire a suitable signal-to-noise ratio $(\mathrm{S} / \mathrm{N})$ in a reasonable amount of $H S T$ time. The properties of these two stars are summarized below.

\subsection{Ross 825}

Ross 825 was first identified as a high proper motion star by Ross (1929), who estimated a total proper motion of 0 ". $48 \mathrm{yr}^{-1}$. Bidelman (1985) compiled spectral classifications determined by G. P. Kuiper and published a spectral type of K3 for Ross 825 . Ross 825 was also noted as a close visual double in that work. The RV of Ross 825 was first measured in Carney \& Latham (1987), who determined a value of $-340.68 \mathrm{~km} \mathrm{~s}^{-1}$.

Gaia observations (Gaia Collaboration et al. 2016, 2018) of Ross 825 confirmed the binary nature of this source (separation $\approx 1$ !" 6 ) and its RV $\left(-340.16 \pm 0.67 \mathrm{~km} \mathrm{~s}^{-1}\right)$. It is also resolved as a binary in the Space Telescope Imaging Spectrograph (STIS) acquisition images acquired through this investigation. Stassun et al. (2018) provided an effective temperature estimate ( $\left.T_{\text {eff }}\right)$ of $4680 \pm 177 \mathrm{~K}$, which corresponds to a spectral type between K3 and K4 (Pecaut \& Mamajek 2013), consistent with its measured spectral type. Schuster et al. (2006) provided $u b v y-\mathrm{H} \beta$ photometry of Ross 825 and determined a photometric metallicity of $[\mathrm{Fe} / \mathrm{H}]=-1.28$ based on empirical relations. An $[\mathrm{Fe} / \mathrm{H}]$ value of -1.28 implies an age $>10 \mathrm{Gyr}$ according to the models of Dotter et al. (2017), which is supported by its large absolute radial velocity. Relevant properties of Ross 825 are summarized in Table 1.

\subsection{Ross 1044}

Ross (1939) identified Ross 1044 as a high proper motion star with a total proper motion of 1 !' $27 \mathrm{yr}^{-1}$. A spectral type of K7 was first published for this star in Bidelman (1985). Newton et al. (2014) determined a near-infrared spectral type of M2 for Ross 1044 . We determine a spectral type of M0 by comparing
Table 2

$H S T /$ STIS Observations

\begin{tabular}{lcc}
\hline \hline Object & $\begin{array}{c}\text { Obs. Dates } \\
\text { UTC }\end{array}$ & $\begin{array}{c}\text { Exposure Time } \\
(\mathrm{s})\end{array}$ \\
\hline Ross 825 & 2018 Jul 30, 2018 Aug 3 & 17079 \\
Ross 1044 & 2018 Nov 26, 2018 Nov 30 & 15186 \\
\hline
\end{tabular}

the publicly available optical spectrum from the Palomar/MSU (Michigan State University) survey (Reid et al. 1995) to Sloan Digital Sky Survey (SDSS) standards (Bochanski et al. 2010). Ross 1044 was found to have a large absolute RV in the Palomar/MSU nearby star spectroscopic survey $\left(-159.1 \mathrm{~km} \mathrm{~s}^{-1}\right.$; Reid et al. 1995). This RV was confirmed in Newton et al. (2014) $\left(-174 \pm 5 \mathrm{~km} \mathrm{~s}^{-1}\right)$ and Gaia DR2 $\left(-169.55 \pm 1.80 \mathrm{~km} \mathrm{~s}^{-1}\right)$. Ross 1044 was also found to have a low metallicity $([\mathrm{Fe} / \mathrm{H}]=-1.01 \pm 0.21$; Newton et al. 2014), which is consistent with an age $>10 \mathrm{Gyr}$ (Dotter et al. 2017). This star also showed no clear signature of $\mathrm{H} \alpha$ either in emission or absorption in the optical spectrum from the Palomar/MSU survey (Reid et al. 1995). Note that while Ross 1044 has a measured RV in Gaia DR2, there is no parallax available. This is likely because Ross 1044 has a visibility_periods_used value of 5 in the Gaia DR2 catalog, which does not satisfy the criterion needed for a five-parameter solution in Equation (11) of Lindegren et al. (2018). Two parallax measurements exist in the literature: $47.0 \pm 4.1$ mas (van Altena et al. 1995) and $26.9 \pm 8.4$ mas (Finch \& Zacharias 2016). The "quality of interagreement" is listed as "Poor" in van Altena et al. (1995), and we therefore adopt the Finch \& Zacharias (2016) parallax for this work. Relevant properties of Ross 1044 are summarized in Table 1.

\section{Observations}

We observed Ross 825 and Ross 1044 during HST Cycle 25 with STIS on board HST (PID \#15286). For these observations, STIS is preferred over the Cosmic Origins Spectrograph (COS) because $\operatorname{COS}$ is a slitless spectrograph, which results in heavy contamination in the Ly $\alpha$ region from geocoronal emission. We employed the G140M grating $(R \sim 11400)$ with a $52^{\prime \prime} \times 0$." 1 slit. For each observation, we set the central wavelength to $1222 \AA$. Observing dates and total exposure times are provided in Table 2.

For several of the visits for each object, the HST calstis pipeline failed to locate the correct location of the star. For both stars, we determined trace positions manually and reran the calstis pipeline for every visit. For Ross 825 , one visit was especially noisy and a trace position could not be determined. These data are not included in the final spectrum. Final spectra 


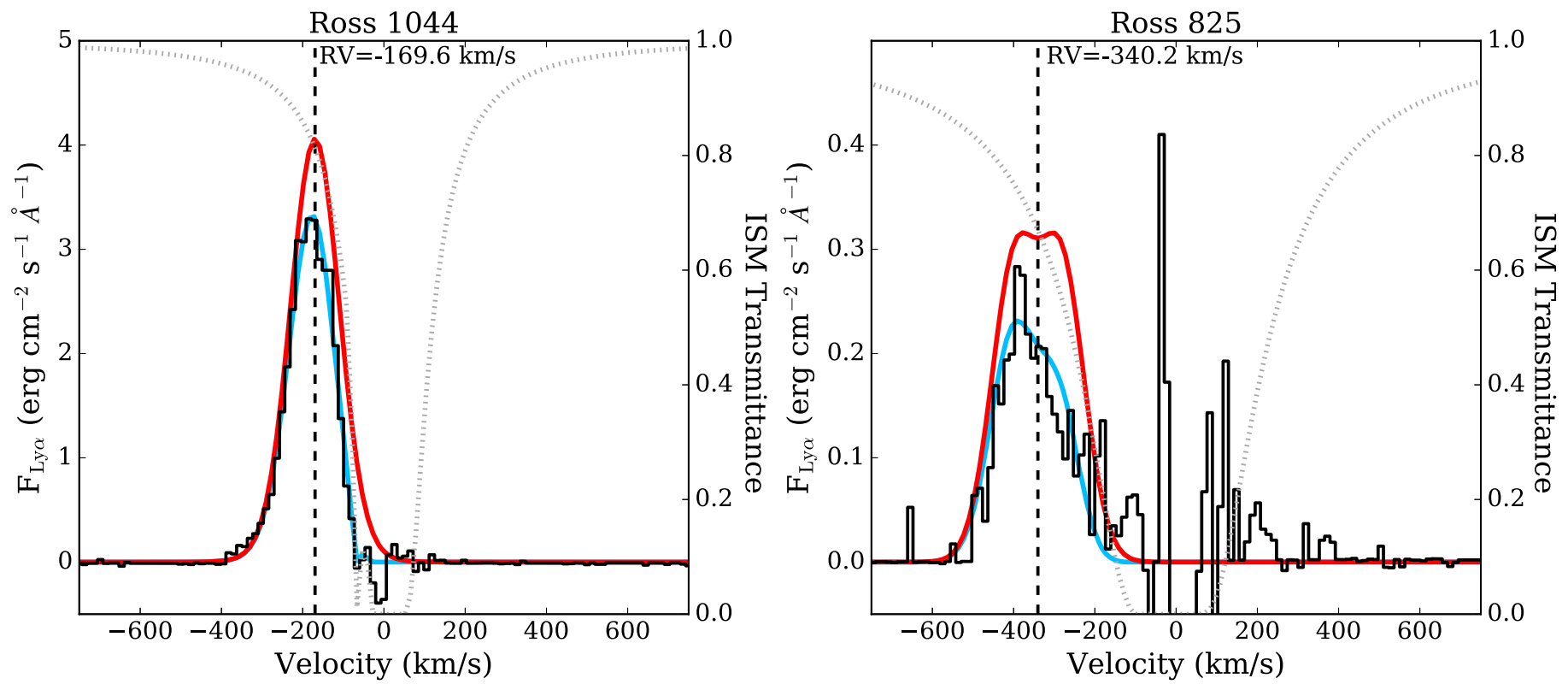

Figure 1. HST/STIS spectra (black lines) for Ross 1044 (left) and Ross 825 (right). The best-fitting model is show in blue, with the resulting intrinsic Ly $\alpha$ flux shown in red. The ISM transmittance curve is shown as a dotted line.

(The data used to create this figure are available.)

were produced by coadding the spectra for each visit together for each star and are shown in Figure 1. While the binary nature of Ross 825 was seen in the STIS acquisition images, the Ly $\alpha$ spectrum comes solely from the primary component of this system. All subsequent analysis of Ross 825 corresponds exclusively to the primary object of this pair.

\section{Analysis}

\subsection{Ly Line Profiles}

For both Ross 825 and Ross 1044, the majority of their Ly $\alpha$ emission is directly measured with our STIS observations. However, neither target is immune to intervening ISM absorption. For this reason, ISM modeling is necessary to retrieve the intrinsic $\operatorname{Ly} \alpha$ flux for both stars. Following Bourrier et al. (2015), we model the ISM using Voigt profiles for both atomic hydrogen and deuterium. We fix the D I/H I ratio to $1.5 \times 10^{-5}$ (Hébrard \& Moos 2003; Linsky et al. 2006). We fix the RV of the Ly $\alpha$ emission to the stellar RV measurements given in Table 1. Free parameters then include the intrinsic Ly $\alpha$ flux, the column density of hydrogen, and the $\mathrm{RV}$ of the intervening ISM. While there may be multiple ISM components along the line of sight to each star, we model them as a single component.

Whether or not intrinsic Ly $\alpha$ line profiles contain a selfreversal, making them single- or double-peaked, is a large source of uncertainty for Ly $\alpha$ reconstructions, leading to flux differences as large as 30\% (Youngblood et al. 2016). For strong lines, such as $\operatorname{Ly} \alpha$, the emission in the wings and the core of the line can form in different atmospheric layers, leading to self-reversals in the core (Peacock et al. 2019). While the Sun shows a self-reversal in its Ly $\alpha$ core, Youngblood et al. (2016) argue that M dwarf Ly $\alpha$ profiles should be single-peaked because M dwarf Mg II profiles show signs of self-absorption. However, we note that the presence of reversals in observed $\mathrm{Mg}$ II line cores of $\mathrm{M}$ dwarfs is also a consequence of spectral resolution. Fontenla et al. (2016) presented a STIS/E230H spectrum of the M2 star GJ 832, which shows clear signs of reversals in its $\mathrm{Mg}$ II line cores. These reversals are not seen in the MUSCLES NUV spectra (see Figure 5 of France et al. 2016), which were taken with the COS/G230L setting, which has $\sim 1$ order of magnitude less spectral resolution than STIS/E230H.

Our STIS spectra have resolutions similar to COS/G230L. All models are convolved with the STIS line-spread function to compare to observations. For Ross 1044, the Ly $\alpha$ line shows a clear Gaussian shape, and the peak of the Ly $\alpha$ emission occurs at the same RV as the stellar RV measurement. Ross 825, on the other hand, has $\mathrm{Ly} \alpha$ emission peaking approximately $60 \mathrm{~km} \mathrm{~s}^{-1}$ away from the measured stellar RV, suggestive of a double-peaked profile. We therefore model our M dwarf target (Ross 1044) without a self-reversal and explore models with and without self-reversals for our K dwarf target (Ross 825). Note that a similar RV offset between peak Ly $\alpha$ emission and the stellar RV is seen for Kepler-444 (spectral type =K1), which has an RV of $-121.4 \mathrm{~km} \mathrm{~s}^{-1}$ (Dupuy et al. 2016). Bourrier et al. (2017a) found that the Ly $\alpha$ emission for Kepler444 is best fit by a double-peaked profile.

For Ross 1044 we use a model consisting of two Gaussians, one strong and narrow and the other weaker and broad, following Youngblood et al. (2016). We also fit the Ross 825 data with two Gaussians, though the second Gaussian in this case is subtracted from the first to account for the presence of a self-reversal in the line core. We find posterior distributions for each model parameter using the emcee package (ForemanMackey et al. 2013). Fore each fit, we run 1000 walkers with 1000 steps, treating the first 300 steps as the burn in sample. For both objects, we also performed a fit with a single Gaussian, and the two-Gaussian solution is preferred in both cases according to the Bayesian information criterion (BIC). For Ross 825, we also attempted a three-Gaussian fit-broad and narrow profiles as with Ross 1044 and a third component 

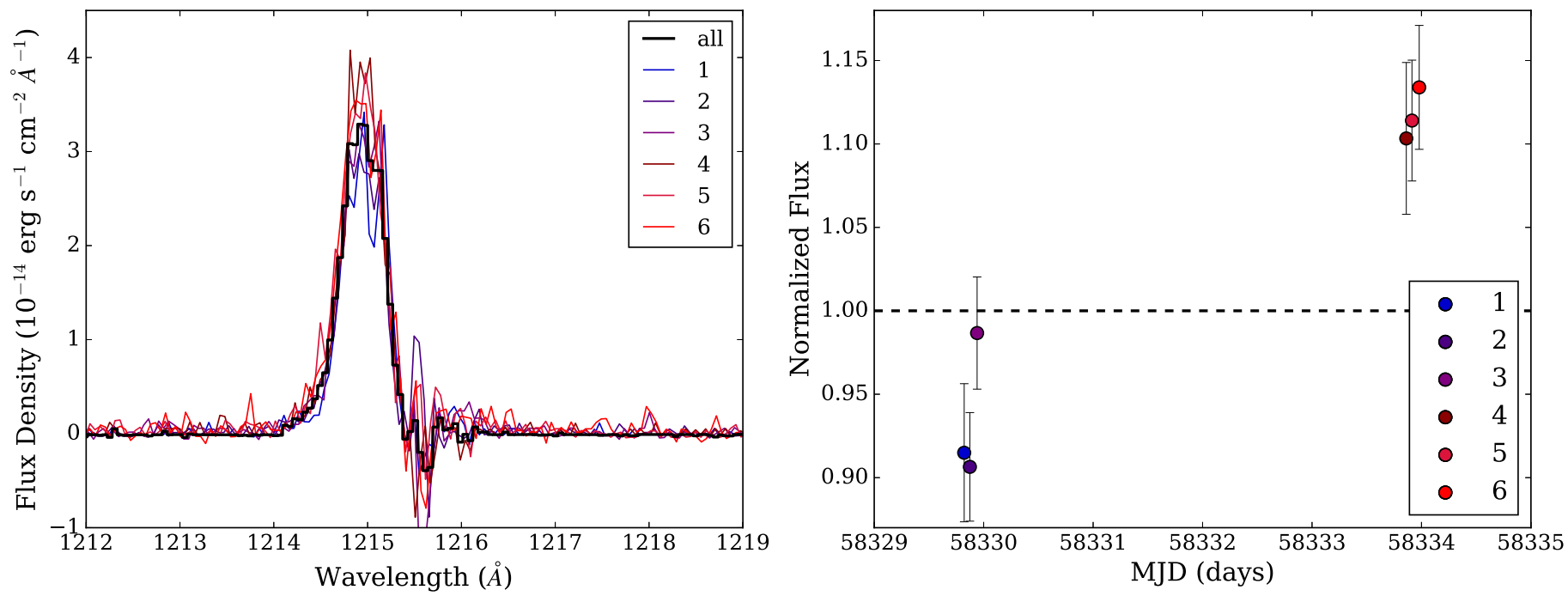

Figure 2. Left: STIS Ly $\alpha$ spectra of Ross 1044 for all six visits compared to the final coadded spectrum. Right: Ly $\alpha$ flux variability of Ross 1044 . All points are normalized to the flux of the final coadded spectrum.

Table 3

emcee Best-fit Model Parameters

\begin{tabular}{|c|c|c|c|c|}
\hline \multirow{2}{*}{ Model Parameter } & \multicolumn{2}{|c|}{ Ross 1044} & \multicolumn{2}{|c|}{ Ross 825} \\
\hline & Prior & Value & Prior & Value \\
\hline Amplitude $1\left(10^{-14} \mathrm{erg} \mathrm{s}^{-1} \mathrm{~cm}^{-2} \AA^{-1}\right)$ & $\mathcal{U}(0,10)$ & $3.75_{-1.46}^{+0.34}$ & $\mathcal{U}(0,10)$ & $1.86_{-0.96}^{+1.82}$ \\
\hline Amplitude $2^{\mathrm{a}}\left(10^{-14} \mathrm{erg} \mathrm{s}^{-1} \mathrm{~cm}^{-2} \AA^{-1}\right)$ & $\mathcal{U}(0,5)$ & $1.55_{-0.96}^{+1.82}$ & $\mathcal{U}(0,5)$ & $0.31_{-0.20}^{+0.61}$ \\
\hline $\operatorname{Sigma} 2^{\mathrm{a}}\left(\mathrm{km} \mathrm{s}^{-1}\right)$ & $\mathcal{U}(0,25)$ & $0.35_{-0.12}^{+1.57}$ & $\mathcal{U}(0,5)$ & $0.24_{-0.03}^{+0.17}$ \\
\hline $\mathrm{RV}_{\mathrm{ISM}}\left(\mathrm{km} \mathrm{s}^{-1}\right)$ & $\mathcal{U}(-50,50)$ & $16.32_{-3.93}^{+5.18}$ & $\mathcal{U}(-50,50)$ & $-13.39_{-24.77}^{+30.63}$ \\
\hline
\end{tabular}

Notes.

${ }^{\text {a }}$ The Gaussian described by Amplitude 2 and Sigma 2 was added to the first Gaussian in the case of Ross 1044, and subtracted in the case of Ross 825 .

${ }^{\mathrm{b}}$ Range of H I column densities taken from Redfield \& Linsky (2008).

subtracted out. Again, the BIC prefers the two-Gaussian model compared to the three-Gaussian case. Our model fits imply that our STIS observed spectra recovered $\sim 74 \%$ and $\sim 63 \%$ of the total intrinsic Ly $\alpha$ flux for Ross 1044 and Ross 825, respectively. These percentages are significantly higher than that typically seen for field $\mathrm{M}$ dwarfs, which is usually $\lesssim 30 \%$ (Youngblood et al. 2017). The best-fitting profiles are shown in Figure 1 with the best-fit parameters given in Table 3 and the resulting Ly $\alpha$ fluxes are provided in Table 4.

\section{Discussion}

\subsection{Lya Variability of Ross 1044}

Ross 1044 has sufficient $\mathrm{S} / \mathrm{N}$ in each of the six visits to investigate for variability in the strength of the Ly $\alpha$ line. In Figure 2, we plot the the individual spectra from each visit, as well as our combined final spectrum. While the general shape and flux are similar for each of the six visits, the total flux for the first three visits is less than that from the last three visits. We quantify this by summing up the total flux in each visit, then normalizing to the total flux from the final combined spectrum, with uncertainties calculated in a Monte Carlo fashion. We find that the total flux varied by 20\% between the smallest and largest flux values measured. Note that there exists an STIS "breathing" effect, which can cause variability within HST orbits (Brown et al. 2001; Sing et al. 2008). This effect should be mitigated by the use of spectra averaged over each visit. The Ly $\alpha$ variability of Ross 1044 is consistent with that seen in the Ly $\alpha$ line of GJ 1132, where a variability up to $22 \%$ is seen (Waalkes et al. 2019), and Kepler-444, where variability of $20 \%-40 \%$ is seen between visits (Bourrier et al. 2017a). This is also consistent with the variability seen in other UV spectral lines in Loyd \& France (2014), where variability from $1 \%-41 \%$ is measured. The number and cadence of our observations prevent us from determining the cause of Ross 1044's variability (e.g., rotational modulations, flares). A dedicated monitoring campaign could pinpoint the cause of this variability.

\subsection{Ly $\alpha$ Correlations}

Correlating intrinsic Ly $\alpha$ fluxes with stellar properties gives us insight into the behavior of high-energy emission of stars in various evolutionary and temperature regimes. Relationships between stellar properties and $\mathrm{Ly} \alpha$ flux values can also provide a powerful means to predict $\operatorname{Ly} \alpha$ fluxes for stars without $\operatorname{Ly} \alpha$ observations. 
Table 4

Properties of All Stars with Intrinsic Ly $\alpha$ Fluxes

\begin{tabular}{|c|c|c|c|c|c|c|c|c|c|c|c|c|c|}
\hline $\begin{array}{l}\text { R.A. } \\
\left({ }^{\circ}\right)\end{array}$ & $\begin{array}{c}\text { Decl. } \\
\left({ }^{\circ}\right)\end{array}$ & $\begin{array}{l}\text { Other } \\
\text { Name }\end{array}$ & $\begin{array}{l}\text { Spectral } \\
\text { Type }\end{array}$ & References & $\begin{array}{l}T_{\text {eff }} \\
(\mathrm{K})\end{array}$ & References & $\begin{array}{c}\text { Radius } \\
R_{\odot}\end{array}$ & References & $\begin{array}{l}\mathrm{Plx}^{\mathrm{a}} \\
(\mathrm{mas})\end{array}$ & $\begin{array}{c}\mathrm{Ly} \alpha \\
\left(\mathrm{erg} \mathrm{cm}^{-2} \mathrm{~s}^{-1}\right)\end{array}$ & References & $\begin{array}{l}\text { Age } \\
\text { (Gyr) }\end{array}$ & $\overline{\text { References }}$ \\
\hline \multicolumn{14}{|l|}{ New } \\
\hline 317.823653042 & +33.524935535 & Ross 825 & $\mathrm{~K} 3$ & 1 & $4680 \pm 177$ & 19 & $0.57_{-0.07}^{+0.15}$ & 15 & $10.17 \pm 0.08$ & $\left(2.65_{-0.76}^{+0.38}\right) \times 10^{-15}$ & 3 & $>10$ & 3 \\
\hline 225.848498243 & +03.785579277 & Ross 1044 & M0 & 3 & $3754 \pm 95$ & 25 & $0.380 \pm 0.029$ & 25 & $26.9 \pm 8.4$ & $\left(2.54_{-0.11}^{+0.14}\right) \times 10^{-14}$ & 3 & $>10$ & 3 \\
\hline \multicolumn{14}{|l|}{ Old } \\
\hline 067.695503172 & +16.148537247 & SAO 93981 & F2 & 8 & $6567 \pm 49$ & 26 & $1.474_{-0.047}^{+0.058}$ & 27 & $22.12 \pm 0.06$ & $(1.41 \pm 0.28) \times 10^{-12}$ & 63 & $1.16 \pm 0.82$ & 38 \\
\hline$\cdots$ & $\ldots$ & Procyon & F5IV-V & 7 & $6648 \pm 71$ & 21 & $2.13 \pm 0.07$ & 19 & $284.56 \pm 1.26$ & $(1.47 \pm 0.03) \times 10^{-10}$ & 65 & $1.97_{-0.23}^{+0.19}$ & 21 \\
\hline 066.577555282 & +21.470267340 & SAO 76609 & F8V & 9 & $6376 \pm 80$ & 23 & $1.30 \pm 0.02$ & 15 & $20.67 \pm 0.07$ & $(2.70 \pm 0.54) \times 10^{-13}$ & 63 & $0.63 \pm 0.05$ & 41 \\
\hline 076.377476718 & -57.472196938 & $\zeta$ Dor & F9V & 4 & $6147 \pm 53$ & 21 & $1.07_{-0.07}^{+0.02}$ & 15 & $86.02 \pm 0.15$ & $(7.99 \pm 1.60) \times 10^{-12}$ & 63 & $0.68 \pm 0.47$ & 38 \\
\hline 184.446711916 & -36.093975066 & HR 4657 & F9V & 4 & $6258 \pm 39$ & 21 & $1.096 \pm 0.033$ & 27 & $44.74 \pm 0.81$ & $(1.28 \pm 0.26) \times 10^{-12}$ & 63 & $1.8 \pm 0.5$ & 37 \\
\hline 330.795021930 & +18.884241930 & V376 Peg & F9V & 9 & $6071 \pm 20$ & 22 & $1.18 \pm 0.02$ & 15 & $20.68 \pm 0.05$ & $(1.50 \pm 0.30) \times 10^{-13}$ & 63 & $3.83_{-0.70}^{+0.98}$ & 22 \\
\hline 066.900016757 & +15.589088762 & V993 Tau & G0 & 8 & $6197 \pm 94$ & 23 & $1.27_{-0.02}^{+0.03}$ & 15 & $20.93 \pm 0.07$ & $(5.90 \pm 1.18) \times 10^{-13}$ & 63 & $0.63 \pm 0.05$ & 41 \\
\hline 088.594850554 & +20.275864770 & $\chi$ Ori & G0V & 4 & $5898 \pm 25$ & 17 & $0.9791 \pm 0.0091$ & 17 & $113.12 \pm 0.32$ & $(1.27 \pm 0.25) \times 10^{-11}$ & 63 & $0.3 \pm 0.1$ & 37 \\
\hline 238.171482022 & +42.454228071 & $\chi$ Her & G0 & 1 & $5890 \pm 53$ & 28 & $1.7090 \pm 0.0200$ & 29 & $63.16 \pm 0.15$ & $(2.04 \pm 0.41) \times 10^{-12}$ & 63 & $6.85_{-0.50}^{+0.42}$ & 22 \\
\hline 271.599395673 & -36.019748261 & HR 6748 & G0V & 4 & $5932 \pm 80$ & 23 & $0.98_{-0.04}^{+0.01}$ & 15 & $58.13 \pm 0.12$ & $(3.73 \pm 0.75) \times 10^{-12}$ & 63 & $0.44 \pm 0.19$ & 40 \\
\hline$\ldots$ & $\ldots$ & Sun & G2V & $\ldots$ & 5778 & $\ldots$ & 1.0 & $\ldots$ & $\ldots$ & $5.95-9.15^{\mathrm{b}}$ & 65 & $4.566 \pm 0.005$ & 66 \\
\hline$\ldots$ & $\ldots$ & $\alpha$ Cen A & $\mathrm{G} 2 \mathrm{~V}$ & 4 & $5793 \pm 7$ & 17 & $1.2329 \pm 0.0037$ & 17 & $754.81 \pm 4.11$ & $(1.01 \pm 0.20) \times 10^{-10}$ & 63 & $5.3 \pm 0.3$ & 34 \\
\hline 112.676660493 & -37.339135172 & HR 2882 & $\mathrm{G} 2 \mathrm{~V}$ & 4 & $5830 \pm 76$ & 22 & $0.93_{-0.01}^{+0.02}$ & 15 & $45.93 \pm 0.03$ & $(2.76 \pm 0.55) \times 10^{-12}$ & 63 & $0.35 \pm 0.07$ & 40 \\
\hline 168.133469825 & +35.813428317 & HR 4345 & $\mathrm{G} 2$ & 1 & $5906 \pm 29$ & 28 & $0.99 \pm 0.03$ & 30 & $44.14 \pm 0.04$ & $(2.09 \pm 0.42) \times 10^{-12}$ & 63 & $0.45 \pm 0.02$ & 40 \\
\hline 049.841563537 & +03.370603059 & $\kappa$ Cet & G4 & 1 & $5723 \pm 76$ & 17 & $0.9193 \pm 0.0247$ & 17 & $109.34 \pm 0.31$ & $(8.44 \pm 1.70) \times 10^{-12}$ & 63 & $0.6 \pm 0.2$ & 22 \\
\hline 129.458268090 & -06.806694022 & SAO 136111 & G5V & 7 & $5836 \pm 45$ & 28 & $1.00 \pm 0.02$ & 15 & $41.08 \pm 0.04$ & $(1.34 \pm 0.27) \times 10^{-12}$ & 63 & $0.51 \pm 0.14$ & 40 \\
\hline 093.438506782 & -23.861458285 & HR 2225 & G6.5V & 4 & $5651 \pm 43$ & 28 & $0.890 \pm 0.04$ & 18 & $59.76 \pm 0.02$ & $(3.45 \pm 0.69) \times 10^{-12}$ & 63 & $0.28-0.36$ & 39 \\
\hline 199.596454977 & -18.315774489 & $61 \mathrm{Vir}$ & G7V & 4 & $5538 \pm 13$ & 18 & $0.987 \pm 0.005$ & 18 & $117.57 \pm 0.24$ & $(1.69 \pm 0.34) \times 10^{-12}$ & 63 & $9.41_{-3.15}^{+1.31}$ & 22 \\
\hline 222.848032870 & +19.100275013 & $\xi$ Boo A & G7V & 7 & $5483 \pm 32$ & 17 & $0.8627 \pm 0.0107$ & 17 & $148.52 \pm 0.24$ & $(1.85 \pm 0.37) \times 10^{-11}$ & 63 & $0.2 \pm 0.1$ & 37 \\
\hline 001.655138540 & +29.020738353 & HR 8 & G8V & 7 & $5327 \pm 39$ & 17 & $0.9172 \pm 0.0090$ & 17 & $72.58 \pm 0.05$ & $(4.77 \pm 0.95) \times 10^{-12}$ & 63 & $0.3 \pm 0.1$ & 22 \\
\hline 220.129108915 & -16.209576638 & SAO 158720 & G8V & 4 & $5574 \pm 50$ & 23 & $0.87_{-0.04}^{+0.02}$ & 15 & $42.09 \pm 0.04$ & $(1.44 \pm 0.29) \times 10^{-12}$ & 63 & $0.62 \pm 0.07$ & 40 \\
\hline 321.170945318 & -68.227107394 & SAO 254993 & G8V & 4 & $5480 \pm 80$ & 23 & $0.87 \pm 0.01$ & 15 & $48.06 \pm 0.34$ & $(2.47 \pm 0.49) \times 10^{-12}$ & 63 & $0.33 \pm 0.08$ & 40 \\
\hline 026.009302877 & -15.933798651 & $\tau$ Cet & $\mathrm{G} 8.5 \mathrm{~V}$ & 4 & $5290 \pm 39$ & 17 & $0.8154 \pm 0.0122$ & 17 & $277.52 \pm 0.52$ & $(9.99 \pm 2.0) \times 10^{-12}$ & 63 & $5.6 \pm 1.2$ & 37 \\
\hline 201.437998838 & +56.970541983 & SAO 28753 & G9V & 7 & $5308 \pm 36$ & 28 & $0.84 \pm 0.03$ & 30 & $46.17 \pm 0.02$ & $(1.62 \pm 0.32) \times 10^{-12}$ & 63 & $0.33 \pm 0.10$ & 40 \\
\hline 300.182122282 & +22.709775924 & HD 189733 & $\mathrm{~K} 2 \mathrm{~V}$ & 7 & $5019 \pm 23$ & 44 & $0.76 \pm 0.01$ & 44 & $50.57 \pm 0.03$ & $(7.48 \pm 1.50) \times 10^{-13}$ & 65 & $6.4_{-4.2}^{+4.8}$ & 30 \\
\hline 085.334752342 & +53.478804107 & HR 1925 & $\mathrm{KOV}$ & 7 & $5243 \pm 32$ & 28 & $0.850 \pm 0.050$ & 18 & $81.43 \pm 0.05$ & $(4.57 \pm 0.91) \times 10^{-12}$ & 63 & $0.5 \pm 0.1$ & 22 \\
\hline 063.808266649 & -07.667602660 & 40 Eri A & $\mathrm{K} 0.5 \mathrm{~V}$ & 4 & $5147 \pm 14$ & 18 & $0.805 \pm 0.004$ & 18 & $198.57 \pm 0.51$ & $(6.93 \pm 1.39) \times 10^{-12}$ & 63 & $11.76_{-5.19}^{+1.92}$ & 22 \\
\hline 143.181619543 & +26.987467661 & DX Leo & K1V & 4 & $5315 \pm 35$ & 28 & $0.81 \pm 0.02$ & 15 & $55.32 \pm 0.06$ & $(2.31 \pm 0.46) \times 10^{-12}$ & 63 & $0.25 \pm 0.05$ & 43 \\
\hline 168.637658369 & +25.710596159 & HD 97658 & $\mathrm{~K} 1$ & 7 & $5157 \pm 29$ & 28 & $0.72 \pm 0.02$ & 30 & $46.35 \pm 0.05$ & $\left(9.1_{-4.5}^{+9.9}\right) \times 10^{-13}$ & 59 & $9.7 \pm 2.8$ & 44 \\
\hline 271.365865680 & +02.494347134 & 70 Oph A & K1 & 1 & $5407 \pm 52$ & 18 & $0.831 \pm 0.004$ & 18 & $195.22 \pm 0.10$ & $(2.14 \pm 0.43) \times 10^{-11}$ & 63 & $1.3 \pm 0.3$ & 37 \\
\hline 289.752832267 & +41.631884026 & Kepler-444 & K1 & 1 & $5053 \pm 45$ & 28 & $0.748 \pm 0.006$ & 24 & $27.41 \pm 0.03$ & $(5.47 \pm 1.77) \times 10^{-14}$ & 60 & $11.23_{-0.99}^{+0.91}$ & 33 \\
\hline 043.135621621 & -12.770528318 & EP Eri & $\mathrm{K} 1.5 \mathrm{~V}$ & 4 & $5167 \pm 44$ & 21 & $0.79_{-0.02}^{+0.04}$ & 15 & $96.54 \pm 0.04$ & $(6.05 \pm 1.21) \times 10^{-12}$ & 63 & $0.2 \pm 0.1$ & 22 \\
\hline$\ldots$ & $\ldots$ & $\alpha$ Cen B & K2IV & 4 & $5232 \pm 8$ & 17 & $0.8761 \pm 0.0029$ & 17 & $796.92 \pm 25.90$ & $(1.50 \pm 0.30) \times 10^{-10}$ & 63 & $5.3 \pm 0.3$ & 34 \\
\hline 004.587759006 & +30.955409811 & PW And & $\mathrm{K} 2 \mathrm{~V}$ & 11 & $4796 \pm 80$ & 23 & $0.72_{-0.02}^{+0.01}$ & 15 & $35.29 \pm 0.05$ & $(2.31 \pm 0.46) \times 10^{-12}$ & 63 & $0.15_{-0.02}^{+0.05}$ & 45 \\
\hline 053.228430601 & -09.458171515 & $\epsilon$ Eri & $\mathrm{K} 2 \mathrm{~V}$ & 4 & $5077 \pm 35$ & 18 & $0.735 \pm 0.005$ & 18 & $312.22 \pm 0.47$ & $(6.1 \pm 0.2) \times 10^{-11}$ & 59 & $0.5 \pm 0.1$ & 22 \\
\hline 057.604601528 & +17.246412457 & V471 Tau & $\mathrm{K} 2 \mathrm{~V}$ & 10 & $5291 \pm 167$ & 27 & $0.83 \pm 0.03$ & 31 & $20.96 \pm 0.04$ & $(2.87 \pm 0.57) \times 10^{-12}$ & 63 & $0.63 \pm 0.05$ & 41 \\
\hline 143.105446328 & -11.184489206 & LQ Hya & $\mathrm{K} 2 \mathrm{~V}$ & 12 & $5376 \pm 43$ & 17 & $1.0029 \pm 0.0158$ & 17 & $54.68 \pm 0.07$ & $(4.01 \pm 0.80) \times 10^{-12}$ & 63 & $0.07_{-0.02}^{+0.03}$ & 43 \\
\hline 258.834197764 & -26.606646978 & 36 Oph A & K2 & 1 & $5103 \pm 29$ & 28 & $0.76 \pm 0.02$ & 15 & $167.82 \pm 0.16$ & $(1.42 \pm 0.28) \times 10^{-11}$ & 63 & $1.7 \pm 0.4$ & 22 \\
\hline 349.865586265 & +79.003831692 & V368 Cep & $\mathrm{K} 2 \mathrm{~V}$ & 7 & $5075 \pm 36$ & 28 & $0.76_{-0.01}^{+0.02}$ & 15 & $52.73 \pm 0.03$ & $(2.99 \pm 0.60) \times 10^{-12}$ & 63 & $0.09_{-0.04}^{+0.06}$ & 43 \\
\hline 088.517219072 & -60.023728916 & HD 40307 & $\mathrm{~K} 2.5 \mathrm{~V}$ & 4 & $4925 \pm 71$ & 22 & $0.71 \pm 0.01$ & 15 & $77.27 \pm 0.03$ & $\left(2.0_{-0.9}^{+2.2}\right) \times 10^{-13}$ & 59 & $6.9 \pm 4.0$ & 44 \\
\hline 311.937623890 & -36.595010211 & Speedy Mic & K3V & 13 & $4609 \pm 80$ & 23 & $1.42_{-0.06}^{+0.03}$ & 15 & $14.98 \pm 0.27$ & $(1.85 \pm 0.37) \times 10^{-12}$ & 63 & $0.03 \pm 0.01$ & 40 \\
\hline 330.871402102 & -56.796902312 & $\epsilon$ Ind & K4V & 4 & $4649 \pm 36$ & 21 & $0.732 \pm 0.006$ & 32 & $274.81 \pm 0.25$ & $(3.10 \pm 0.62) \times 10^{-11}$ & 63 & $1.6 \pm 0.2$ & 21 \\
\hline 316.747737594 & +38.763411107 & 61 Cyg A & K5 & 5 & $4361 \pm 17$ & 18 & $0.665 \pm 0.005$ & 35 & $285.95 \pm 0.10$ & $(1.72 \pm 0.34) \times 10^{-11}$ & 63 & $6.0 \pm 1.0$ & 35 \\
\hline 147.782122563 & -43.504816171 & HD 85512 & K6V & 4 & $4455 \pm 80$ & 23 & $0.70_{-0.04}^{+0.02}$ & 15 & $88.62 \pm 0.04$ & $\left(1.2_{-0.5}^{+1.8}\right) \times 10^{-12}$ & 59 & $5.61 \pm 0.61$ & 50 \\
\hline 077.958661296 & -45.043019828 & & sdM1 & 2 & $3570 \pm 80$ & 20 & $0.291 \pm 0.025$ & 20 & $254.23 \pm 0.04$ & $(5.27 \pm 0.26) \times 10^{-13}$ & 20 & $11.5_{-1.5}^{+0.5}$ & 20 \\
\hline
\end{tabular}




\begin{tabular}{|c|c|c|c|c|c|c|c|c|c|c|c|c|c|}
\hline $\begin{array}{l}\text { R.A. } \\
\left({ }^{\circ}\right)\end{array}$ & $\begin{array}{c}\text { Decl. } \\
\left({ }^{\circ}\right)\end{array}$ & $\begin{array}{l}\text { Other } \\
\text { Name }\end{array}$ & $\begin{array}{c}\text { Spectral } \\
\text { Type }\end{array}$ & References & $\begin{array}{l}T_{\text {eff }} \\
(\mathrm{K})\end{array}$ & References & $\begin{array}{c}\text { Radius } \\
R_{\odot}\end{array}$ & References & $\begin{array}{l}\mathrm{Plx}^{\mathrm{a}} \\
(\mathrm{mas})\end{array}$ & $\begin{array}{c}\operatorname{Ly} \alpha \\
\left(\operatorname{erg~cm}^{-2} \mathrm{~s}^{-1}\right)\end{array}$ & References & $\begin{array}{l}\text { Age } \\
(\text { Gyr) }\end{array}$ & References \\
\hline \multicolumn{14}{|c|}{$\begin{array}{c}\text { Kapteyn's } \\
\text { Star }\end{array}$} \\
\hline 311.291136883 & -31.342450016 & $\begin{array}{c}\text { Star } \\
\text { AU Mic }\end{array}$ & M1 & 6 & $3588 \pm 87$ & 16 & $0.698 \pm 0.021$ & 16 & $102.83 \pm 0.05$ & $(1.07 \pm 0.04) \times 10^{-11}$ & 61 & $0.02 \pm 0.01$ & 45 \\
\hline 259.751060918 & -34.997765110 & GJ $667 \mathrm{C}$ & $\mathrm{M} 1.5 \mathrm{~V}$ & 48 & $3472 \pm 73$ & 6 & $0.37 \pm 0.05$ & 6 & $138.02 \pm 0.09$ & $(5.2 \pm 0.9) \times 10^{-13}$ & 59 & $>2$ & 51 \\
\hline 323.391261617 & -49.012516898 & GJ 832 & M2 & 6 & $3522 \pm 60$ & 6 & $0.442 \pm 0.013$ & 16 & $201.40 \pm 0.04$ & $(9.5 \pm 0.6) \times 10^{-13}$ & 59 & 8.4 & 46 \\
\hline 070.735385329 & +18.953357960 & GJ 176 & M2 & 6 & $3679 \pm 77$ & 18 & $0.453 \pm 0.022$ & 18 & $105.56 \pm 0.07$ & $(3.9 \pm 0.2) \times 10^{-13}$ & 59 & $4.0 \pm 0.3$ & 18 \\
\hline 154.898887344 & +19.869815836 & AD Leo & M3 & 6 & $3308 \pm 84$ & 16 & $0.422 \pm 0.013$ & 16 & $201.37 \pm 0.07$ & $(8.07 \pm 0.20) \times 10^{-12}$ & 61 & $0.025-0.3$ & 36 \\
\hline 175.550536327 & +26.703066902 & GJ 436 & M3 & 6 & $3416 \pm 53$ & 18 & $0.455 \pm 0.018$ & 18 & $102.50 \pm 0.09$ & $(2.1 \pm 0.3) \times 10^{-13}$ & 59 & $4.2 \pm 0.3$ & 18 \\
\hline 229.856472500 & -07.722693495 & GJ 581 & M3 & 6 & $3415 \pm 87$ & 16 & $0.330 \pm 0.01$ & 16 & $158.75 \pm 0.05$ & $\left(1.1_{-0.2}^{+0.3}\right) \times 10^{-12}$ & 59 & $4.1 \pm 0.3$ & 18 \\
\hline 258.831399085 & +04.960679473 & GJ 1214 & $\mathrm{M} 4 \mathrm{~V}$ & 49 & $3008 \pm 96$ & 16 & $0.203 \pm 0.008$ & 16 & $68.27 \pm 0.17$ & $\left(1.3_{-0.5}^{+1.4}\right) \times 10^{-14}$ & 59 & $5-10$ & 52 \\
\hline 343.323973712 & -14.266595816 & GJ 876 & M4 & 6 & $3129 \pm 19$ & 18 & $0.376 \pm 0.006$ & 18 & $213.87 \pm 0.08$ & $(3.9 \pm 0.4) \times 10^{-13}$ & 59 & $9.51 \pm 0.58$ & 47 \\
\hline 153.709069589 & -47.154935771 & GJ 1132 & M4 & 1 & $3216 \pm 100$ & 16 & $0.217 \pm 0.008$ & 16 & $79.25 \pm 0.04$ & $\left(2.9_{-0.3}^{+0.4}\right) \times 10^{-14}$ & 64 & $>5.0$ & 62 \\
\hline 341.702962603 & +44.332017083 & EV Lac & M5 & 6 & $3273 \pm 86$ & 16 & $0.341 \pm 0.010$ & 16 & $198.01 \pm 0.04$ & $(2.75 \pm 0.55) \times 10^{-12}$ & 63 & $0.025-0.3$ & 36 \\
\hline 217.393465743 & -62.676182103 & Prox Cen & M5.5 & 14 & $2840 \pm 88$ & 16 & $0.154 \pm 0.005$ & 16 & $768.50 \pm 0.20$ & $(4.37 \pm 0.07) \times 10^{-12}$ & 61 & $5.3 \pm 0.3$ & 34 \\
\hline 346.626391870 & -05.043461802 & Trappist-1 & M8 & 53 & $2550 \pm 55$ & 53 & $0.117 \pm 0.004$ & 53 & $80.45 \pm 0.12$ & $(7.5 \pm 2.3) \times 10^{-15}$ & 53 & $7.6 \pm 2.2$ & 54 \\
\hline \multirow[t]{2}{*}{278.907460881} & +32.994892032 & LSR & M8.5 & 55 & $2578 \pm 39$ & 56 & $0.137 \pm 0.007$ & 57 & $175.82 \pm 0.09$ & $(7.0 \pm 3.5) \times 10^{-15}$ & 58 & $\cdots$ & $\ldots$ \\
\hline & & $\mathrm{J} 1835+3259$ & & & & & & & & & & & \\
\hline
\end{tabular}

Notes.

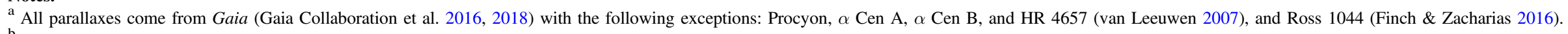

${ }^{b}$ The Ly $\alpha$ flux range given for the Sun encompasses both the active and quiet Sun values given in Linsky et al. (2013).

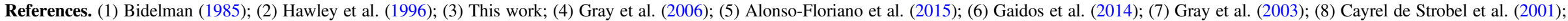

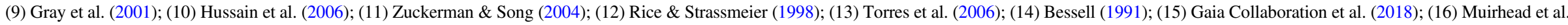

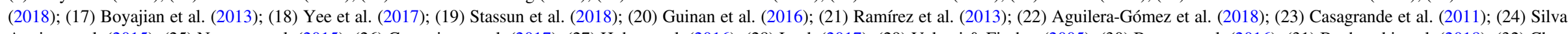

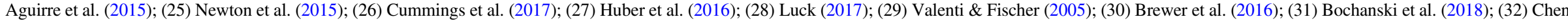

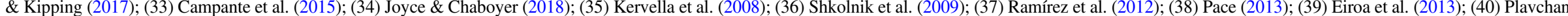

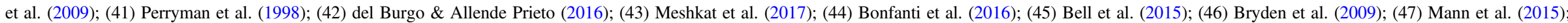

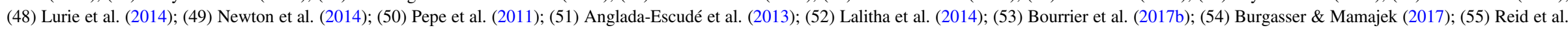

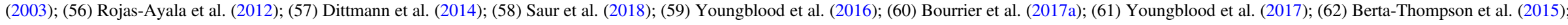
(63) Wood et al. (2005); (64) Waalkes et al. (2019); (65) Linsky et al. (2013); (66) Bahcall et al. (1995). 

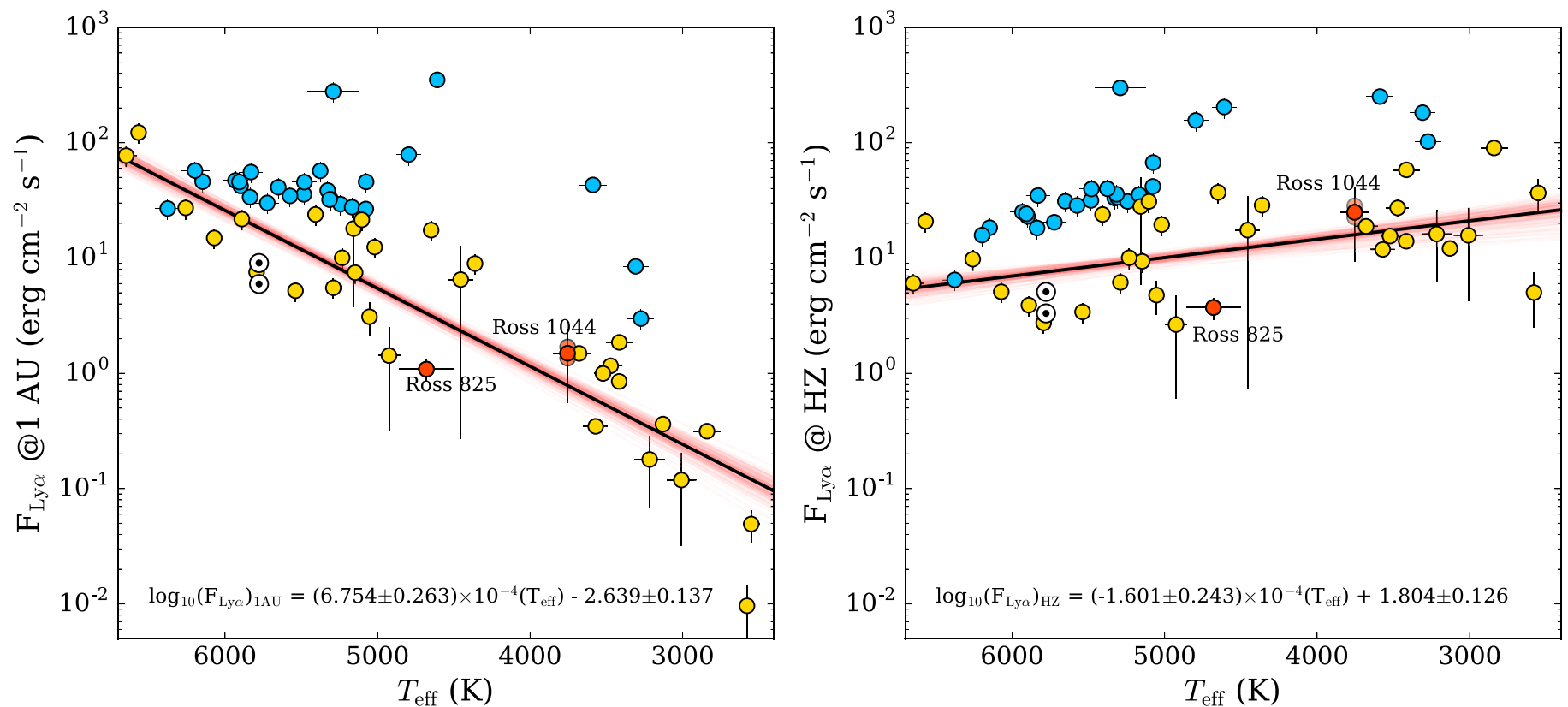

Figure 3. Left: Ly $\alpha$ Flux at 1 au vs. $T_{\text {eff }}$ for all objects in Table 4. Young stars (age $<1 \mathrm{Gyr}$ ) are plotted in light blue, while old stars (age $>1$ Gyr) are plotted in yellow. Ross 825 and Ross 1044 (age $>10 \mathrm{Gyr}$ ) are plotted in red. Two additional symbols are plotted for Ross 1044 representing the maximum and minimum variability determined in Section 5.1. The two solar symbols represent values for the quiet and active Sun from Linsky et al. (2013). The solid black line represents the best fit to the data, with red lines showing individual fits from a Monte Carlo analysis. Right: same as the left panel, but with Ly $\alpha$ fluxes scaled to the distance to the habitable zone for each star.

Table 5

GALEX NUV and Ly $\alpha$ Fluxes for M Dwarfs and Young K Dwarfs

\begin{tabular}{|c|c|c|c|c|c|}
\hline Name & $\begin{array}{l}\text { Spectral } \\
\text { Type }\end{array}$ & $\begin{array}{l}\text { Age } \\
(\mathrm{Gyr})\end{array}$ & $\begin{array}{l}\mathrm{Plx}^{\mathrm{a}} \\
(\mathrm{mas})\end{array}$ & $\begin{array}{c}\mathrm{Ly} \alpha \\
\left(\mathrm{erg} \mathrm{cm}-2 \mathrm{~s}^{-1} \text { ) }\right.\end{array}$ & $\begin{array}{l}\text { NUV } \\
\text { (mag) }\end{array}$ \\
\hline PW And & $\mathrm{K} 2$ & $0.15_{-0.02}^{+0.05}$ & $35.29 \pm 0.05$ & $(2.31 \pm 0.46) \times 10^{-12}$ & $\overline{15.317 \pm 0.012}$ \\
\hline Speedy Mic & K3 & $0.03 \pm 0.01$ & $14.98 \pm 0.27$ & $(1.85 \pm 0.37) \times 10^{-12}$ & $15.469 \pm 0.009$ \\
\hline Kapteyn's Star & sdM1 & $11.5_{-1.5}^{+0.5}$ & $254.23 \pm 0.03$ & $(5.27 \pm 0.26) \times 10^{-13}$ & $19.103 \pm 0.048$ \\
\hline AU Mic & M1 & $0.02 \pm 0.01$ & $102.83 \pm 0.05$ & $(1.07 \pm 0.04) \times 10^{-11}$ & $15.614 \pm 0.010$ \\
\hline GJ 832 & M2 & 8.4 & $201.41 \pm 0.04$ & $(9.5 \pm 0.6) \times 10^{-13}$ & $18.393 \pm 0.036$ \\
\hline AD Leo & M3 & $0.025-0.3$ & $201.37 \pm 0.07$ & $(8.07 \pm 0.20) \times 10^{-12}$ & $15.814 \pm 0.017$ \\
\hline GJ 876 & M4 & $9.51 \pm 0.58$ & $213.87 \pm 0.08$ & $(3.9 \pm 0.4) \times 10^{-13}$ & $20.149 \pm 0.087$ \\
\hline Prox Cen & M5.5 & $5.3 \pm 0.3$ & $768.50 \pm 0.20$ & $(4.37 \pm 0.07) \times 10^{-12}$ & $18.601 \pm 0.086$ \\
\hline
\end{tabular}

Note.

${ }^{\text {a }}$ Parallaxes from Gaia (Gaia Collaboration et al. 2016, 2018), with the exception of Ross 1044 (Finch \& Zacharias 2016).

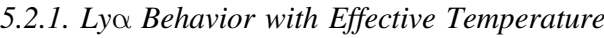

Linsky et al. (2013) showed a correlation between stellar effective temperature $\left(T_{\text {eff }}\right)$ and $\operatorname{Ly} \alpha$ flux. We revisit this relationship here to include additional measurements published since that paper as well as Ross 825 and Ross 1044. These additions increase the entire sample size by $\sim 30 \%$, with 47 Ly $\alpha$ measurements used in Linsky et al. (2013) and 61 used in this work, two of which are new measurements. We also use updated $T_{\text {eff }}$ measurements as well as more precise parallaxes from Gaia DR2 (Gaia Collaboration et al. 2016, 2018). Table 4 lists these properties ( $T_{\text {eff }}$, age, parallax) and intrinsic Ly $\alpha$ fluxes for all stars with Ly $\alpha$ flux measurements. For Kapteyn's star, Guinan et al. (2016) directly measured a Ly $\alpha$ flux of $5.32 \times 10^{-13} \mathrm{erg} \mathrm{s}^{-1}$ $\mathrm{cm}^{-2}$, though no uncertainty is provided for this measurement. We assume an uncertainty of $5 \%$ for this directly measured line.
Figure 3 shows the Ly $\alpha$ flux for each star in Table 4 scaled to 1 au versus their effective temperatures. While Linsky et al. (2013) partition different stellar samples based on their rotation rates, we instead choose to use age because age measurements are more readily available for this sample. We note that determining precise stellar ages is a challenging process, and many different methods can be used depending on the star in question and the data available for that star. All ages used in this work are provided in Table 4. For more information on how a particular age was determined, please refer to the reference provided in the table. There is a clear difference between young $(<1 \mathrm{Gyr})$ and old $(>1 \mathrm{Gyr})$ samples in Figure 3. Ly $\alpha$ emission is typically much stronger from young stars than old stars with similar $T_{\text {eff }}$ values. For old stars, a very clear trend of decreasing Ly $\alpha$ flux strength with decreasing $T_{\text {eff }}$ can be seen. We fit this trend using a least-squares optimization 

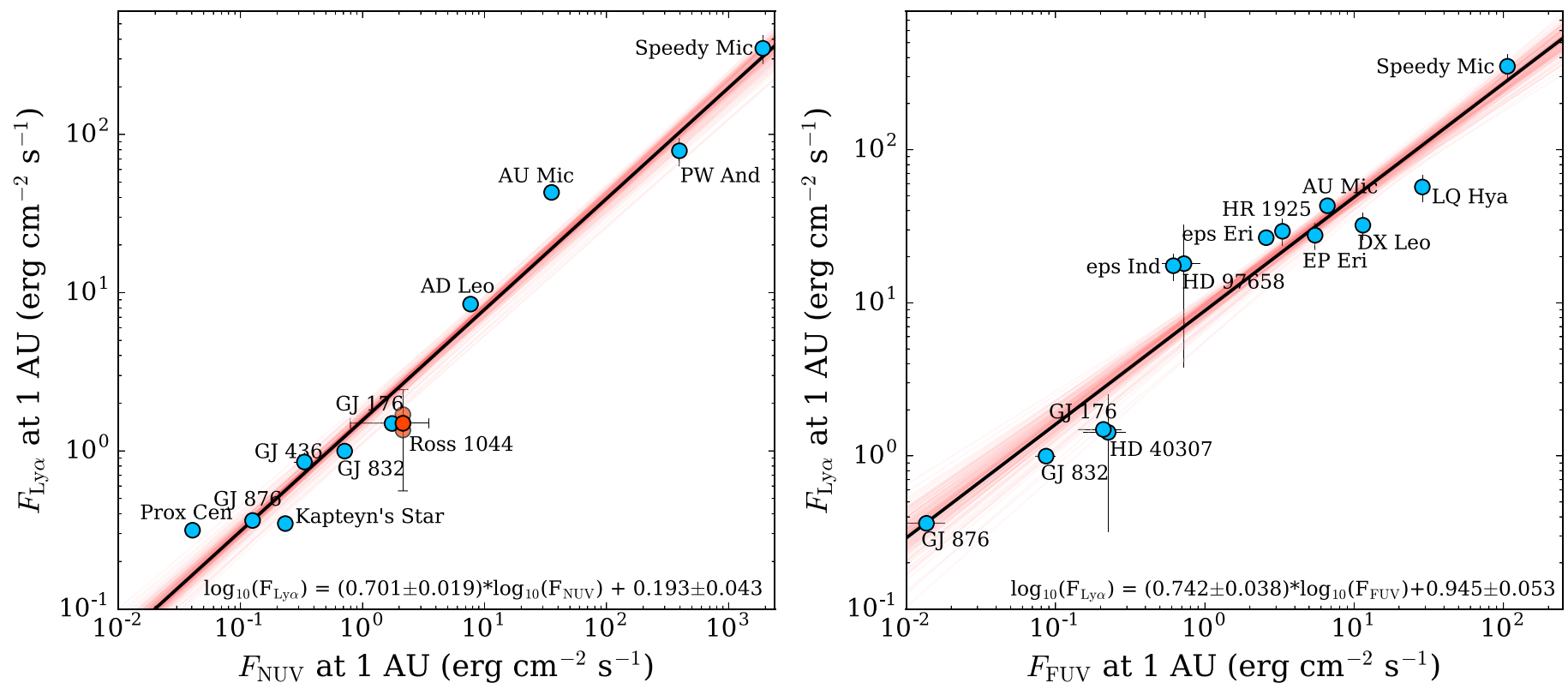

Figure 4. Left: Ly $\alpha$ flux vs. GALEX NUV flux for low-mass stars (light blue symbols) and our new measurement for Ross 1044 (red symbol). Two additional symbols are plotted for Ross 1044 representing the maximum and minimum variability determined in Section 5.1. The solid black line represents the best fit to the data, with red lines showing individual fits from a Monte Carlo analysis. Right: same as left panel, but for GALEX FUV.

Table 6

GALEX FUV and Ly $\alpha$ Fluxes for K and M Dwarfs

\begin{tabular}{|c|c|c|c|c|c|}
\hline Name & $\begin{array}{l}\text { Spectral } \\
\text { Type }\end{array}$ & $\begin{array}{l}\text { Age } \\
(\mathrm{Gyr})\end{array}$ & $\begin{array}{l}\text { Plx } \\
\text { (mas) }\end{array}$ & $\begin{array}{c}\mathrm{Ly} \alpha \\
\left(\mathrm{erg} \mathrm{cm}^{-2} \mathrm{~s}^{-1}\right)\end{array}$ & $\begin{array}{l}\text { FUV } \\
(\mathrm{mag})\end{array}$ \\
\hline DX Leo & K0 & $0.25 \pm 0.05$ & $55.32 \pm 0.06$ & $(2.31 \pm 0.46) \times 10^{-12}$ & $18.188 \pm 0.053$ \\
\hline HR 1925 & K1 & $0.5 \pm 0.1$ & $81.43 \pm 0.05$ & $(4.57 \pm 0.91) \times 10^{-12}$ & $18.695 \pm 0.091$ \\
\hline HD 97658 & K1 & $9.7 \pm 2.8$ & $46.35 \pm 0.05$ & $\left(9.1_{-4.5}^{+9.9}\right) \times 10^{-13}$ & $21.568 \pm 0.308$ \\
\hline EP Eri & $\mathrm{K} 2$ & $0.2 \pm 0.1$ & $96.54 \pm 0.04$ & $(6.05 \pm 1.21) \times 10^{-12}$ & $17.779 \pm 0.018$ \\
\hline$\epsilon$ Eri & $\mathrm{K} 2$ & $0.5 \pm 0.1$ & $312.22 \pm 0.47$ & $(6.1 \pm 0.2) \times 10^{-11}$ & $16.049 \pm 0.023$ \\
\hline LQ Hya & $\mathrm{K} 2$ & $0.07_{-0.02}^{+0.03}$ & $54.68 \pm 0.07$ & $(4.01 \pm 0.80) \times 10^{-12}$ & $17.217 \pm 0.037$ \\
\hline HD 40307 & $\mathrm{~K} 2.5$ & $6.9 \pm 4.0$ & $77.27 \pm 0.03$ & $\left(2.0_{-0.9}^{+2.2}\right) \times 10^{-13}$ & $21.728 \pm 0.346$ \\
\hline Speedy Mic & K3 & $0.03 \pm 0.01$ & $14.98 \pm 0.27$ & $(1.85 \pm 0.37) \times 10^{-12}$ & $18.604 \pm 0.079$ \\
\hline$\epsilon$ Ind & K5 & $1.6 \pm 0.2$ & $274.80 \pm 0.25$ & $(3.10 \pm 0.62) \times 10^{-11}$ & $17.881 \pm 0.047$ \\
\hline AU Mic & M1 & $0.02 \pm 0.01$ & $102.83 \pm 0.05$ & $(1.07 \pm 0.04) \times 10^{-11}$ & $17.436 \pm 0.038$ \\
\hline GJ 832 & M2 & 8.4 & $201.41 \pm 0.04$ & $(9.5 \pm 0.6) \times 10^{-13}$ & $20.687 \pm 0.168$ \\
\hline GJ 176 & M2 & $4.0 \pm 0.3$ & $105.56 \pm 0.07$ & $(3.9 \pm 0.2) \times 10^{-13}$ & $21.130 \pm 0.347$ \\
\hline GJ 876 & M4 & $9.51 \pm 0.58$ & $213.87 \pm 0.08$ & $(3.9 \pm 0.4) \times 10^{-13}$ & $22.560 \pm 0.360$ \\
\hline
\end{tabular}

approach, with uncertainties handled in a Monte Carlo fashion. We find

$$
\begin{aligned}
\log _{10}\left(F_{\mathrm{Ly} \alpha}\right)_{1 \mathrm{au}}= & (6.754 \pm 0.263) \times 10^{-4}\left(T_{\mathrm{eff}}\right) \\
& -2.639 \pm 0.137
\end{aligned}
$$

The rms scatter about the fit is $\sim 0.21$ dex. We also plot in Figure 3 the Ly $\alpha$ flux scaled to the HZ for each target. The distance to the $\mathrm{HZ}$ for each star is calculated using their effective temperatures and radii listed in Table 4 and Equations (4) and (5) of Kopparapu et al. (2013a) and the updated coefficients in Kopparapu et al. (2013b). The HZ distance is taken as the average value between the distances to the moist greenhouse and maximum greenhouse boundaries. While the relation between Ly $\alpha$ flux and $T_{\text {eff }}$ flattens out for this sample, it is notable that the slope of this relation is positive, implying that, in general, the Ly $\alpha$ flux in the $\mathrm{HZ}$ increases with decreasing $T_{\text {eff }}$. The relationship between Ly $\alpha$
$\mathrm{HZ}$ flux and $T_{\text {eff }}$ is

$$
\begin{aligned}
\log _{10}\left(F_{\mathrm{Ly} \alpha}\right)_{\mathrm{HZ}}= & (-1.601 \pm 0.243) \times 10^{-4}\left(T_{\mathrm{eff}}\right) \\
& +1.804 \pm 0.126
\end{aligned}
$$

The rms scatter about the fit is $\sim 0.19$ dex.

\subsubsection{Predicting Ly $\alpha$ Fluxes with GALEX Photometry}

Because $\operatorname{Ly} \alpha$ emission is impossible to measure directly for almost all stars, and even indirect methods, such as reconstructions, require valuable $H S T$ time, correlations between Ly $\alpha$ fluxes and other activity indicators are immensely valuable as predictive tools. Shkolnik et al. (2014) used GALEX photometry and reconstructed Ly $\alpha$ emission profiles from Wood et al. (2005), France et al. (2012, 2013), and Linsky et al. (2013) to show trends between NUV and FUV photometry and the reconstructed Ly $\alpha$ fluxes for $\mathrm{K}$ and $\mathrm{M}$ dwarfs. We update these correlations with precision parallaxes 
from Gaia and new and updated Ly $\alpha$ fluxes from this work, Guinan et al. (2016), Youngblood et al. (2016), and Youngblood et al. (2017). The new NUV relation contains three objects not included in the Shkolnik et al. (2014) relation; Ross 1044, Kapteyn's Star, and GJ 176. We also produce these new correlations using flux at 1 au instead of excess surface flux as in Shkolnik et al. (2014), which avoids any uncertainties from model photospheric flux and radius estimates. Thus for the NUV relation, we only include stars where the photospheric NUV flux is negligible, namely, $\mathrm{M}$ stars (at all ages) and young $\mathrm{K}$ stars (Schneider \& Shkolnik 2018; Richey-Yowell et al. 2019).

Table 5 summarizes all observed Ly $\alpha$ flux measurements for $\mathrm{M}$ stars and young $\mathrm{K}$ stars with NUV detections in GALEX. Note that there are many young $\mathrm{K}$ dwarfs from Table 5 that were observed by GALEX; however, the majority of the sources were saturated (NUV $<15 \mathrm{mag}$ ), and are thus not included here. For objects that have detections from multiple GALEX surveys, the magnitudes presented in Tables 5 and 6 are the weighted average of their flux densities, converted back to magnitude. Figure 4 shows a comparison of the the Ly $\alpha$ flux values versus GALEX NUV flux values at 1 au. We perform a least-squares fit to the data and find the following relation between Ly $\alpha$ flux and GALEX NUV fluxes:

$$
\begin{aligned}
\log _{10}\left(F_{\mathrm{Ly} \alpha}\right)= & (0.701 \pm 0.019) * \log _{10}\left(F_{\mathrm{NUV}}\right) \\
& +0.193 \pm 0.043 .
\end{aligned}
$$

Uncertainties for the fit are determined in a Monte Carlo fashion. The rms scatter about this fit is 0.10 dex.

Some nearby, low-mass stars are too bright for reliable GALEX NUV measurements. For these stars, it may be possible to use FUV fluxes. We plot GALEX FUV fluxes versus Ly $\alpha$ fluxes in Figure 4, with FUV data given in Table 6. All K and M dwarfs with unsaturated GALEX FUV measurements are included in the FUV relation, with the total number of stars used increasing from 10 in Shkolnik et al. (2014) to 13 in this work with the addition of HD 97658, HD 40307, and GJ 176. We perform a fit using the same methods as for GALEX NUV data and find

$$
\begin{aligned}
\log _{10}\left(F_{\mathrm{Ly} \alpha}\right)= & (0.742 \pm 0.038) * \log _{10}\left(F_{\mathrm{FUV}}\right) \\
& +0.945 \pm 0.053
\end{aligned}
$$

The rms scatter about this fit is 0.13 dex. The utility of these correlations can be far-reaching, as GALEX has archived flux measurements for thousands of late- $\mathrm{K}$ and $\mathrm{M}$ dwarfs in the solar neighborhood (e.g., Ansdell et al. 2015; Jones \& West 2016; Kastner et al. 2017; Miles \& Shkolnik 2017; Schneider \& Shkolnik 2018; Richey-Yowell et al. 2019).

\section{Conclusion}

We presented HST/STIS observations of Ross 1044 and Ross 825, two low-mass stars with sufficient RVs to directly observe the majority of each star's intrinsic Ly $\alpha$ flux $(\sim 74 \%$ for Ross 1044 and $\sim 63 \%$ for Ross 825 ). We combined these new Ly $\alpha$ measurements with updated astrometric information and physical parameters to explore the relationship between Ly $\alpha$ flux and $T_{\text {eff. }}$. We also constructed new correlations between Ly $\alpha$ fluxes and GALEX UV photometry.

Astrometric missions such as Gaia will soon reveal numerous additional nearby stars with radial velocities for which such observations are possible. High-RV targets are the only way to directly observe the majority of the Ly $\alpha$ flux from low-mass stars. While the exact RV value needed depends on a target's distance and the ISM properties toward that target, a minimum absolute value of $\sim 100 \mathrm{~km} \mathrm{~s}^{-1}$, corresponding to a wavelength shift of $\approx 0.4 \AA$, is likely necessary. Of the $\sim 73,000$ stars within $50 \mathrm{pc}$ with RV measurements in the Gaia DR2 catalog, only $120(0.16 \%)$ have radial velocities $>100 \mathrm{~km} \mathrm{~s}^{-1}$. Each of these objects are thus invaluable as laboratories for investigating the UV environments of nearby stars.

This work is based on observations made with the NASA/ ESA Hubble Space Telescope, obtained at the Space Telescope Science Institute, which is operated by the Association of Universities for Research in Astronomy, Inc., under NASA contract NAS 5-26555. These observations are associated with program \#15283. Support for program \#15283 was provided by NASA through a grant from the Space Telescope Science Institute, which is operated by the Association of Universities for Research in Astronomy, Inc., under NASA contract NAS 5-26555. A.S. and E.S. appreciate support from NASA/ Habitable Worlds grant NNX16AB62G. T.B. was supported in part by NASA HST grant HST-AR-13911 and NASA Habitable Worlds grant NNX16AB62G. This work is based on observations made with the NASA Galaxy Evolution Explorer. GALEX is operated for NASA by the California Institute of Technology under NASA contract NAS 5-98034. This work has made use of data from the European Space Agency (ESA) mission Gaia (https://www.cosmos.esa.int/ gaia), processed by the Gaia Data Processing and Analysis Consortium (DPAC, https://www.cosmos.esa.int/web/gaia/ dpac/consortium). Funding for the DPAC has been provided by national institutions, in particular the institutions participating in the Gaia Multilateral Agreement.

Software: emcee (Foreman-Mackey et al. 2013).

\section{ORCID iDs}

Adam C. Schneider (iD https://orcid.org/0000-0002-6294-5937 Evgenya L. Shkolnik (i) https://orcid.org/0000-00027260-5821

Travis S. Barman (i) https://orcid.org/0000-0002-7129-3002 R. Parke Loyd (1) https://orcid.org/0000-0001-5646-6668

\section{References}

Aguilera-Gómez, C., Ramírez, I., \& Chanamé, J. 2018, A\&A, 614, A55 Alonso-Floriano, F. J., Morales, J. C., Caballero, J. A., et al. 2015, A\&A, 577, A128

Anglada-Escudé, G., Tuomi, M., Gerlach, E., et al. 2013, A\&A, 556, A126 Ansdell, M., Gaidos, E., Mann, A. W., et al. 2015, ApJ, 798, 41

Arney, G. N. 2019, ApJL, 873, L7

Bahcall, J. N., Pinsonneault, M. H., \& Wasserburg, G. J. 1995, RvMP, 67, 781 Bell, C. P. M., Mamajek, E. E., \& Naylor, T. 2015, MNRAS, 454, 593

Berta-Thompson, Z. K., Irwin, J., Charbonneau, D., et al. 2015, Natur, 527, 204

Bessell, M. S. 1991, AJ, 101, 662

Bidelman, W. P. 1985, ApJS, 59, 197

Bochanski, J. J., Faherty, J. K., Gagné, J., et al. 2018, AJ, 155, 149

Bochanski, J. J., Hawley, S. L., Covey, K. R., et al. 2010, AJ, 139, 2679

Bonfanti, A., Ortolani, S., \& Nascimbeni, V. 2016, A\&A, 585, A5

Bourrier, V., Ehrenreich, D., Allart, R., et al. 2017a, A\&A, 602, A106 Bourrier, V., Ehrenreich, D., \& Lecavelier des Etangs, A. 2015, A\&A, 582, A65 Bourrier, V., Ehrenreich, D., Wheatley, P. J., et al. 2017b, A\&A, 599, L3 Boyajian, T. S., von Braun, K., van Belle, G., et al. 2013, ApJ, 771, 40 Brewer, J. M., Fischer, D. A., Valenti, J. A., et al. 2016, ApJS, 225, 32 Brown, T. M., Charbonneau, D., Gilliland, R. L., et al. 2001, ApJ, 552, 699 Bryden, G., Beichman, C. A., Carpenter, J. M., et al. 2009, ApJ, 705, 1226 Burgasser, A. J., \& Mamajek, E. E. 2017, ApJ, 845, 110 
Campante, T. L., Barclay, T., Swift, J. J., et al. 2015, ApJ, 799, 170 Carney, B. W., \& Latham, D. W. 1987, AJ, 93, 116

Casagrande, L., Schönrich, R., Asplund, M., et al. 2011, A\&A, 530, A138 Cayrel de Strobel, G., Soubiran, C., \& Ralite, N. 2001, A\&A, 373, 159 Chen, J., \& Kipping, D. 2017, ApJ, 834, 17

Cummings, J. D., Deliyannis, C. P., Maderak, R. M., et al. 2017, AJ, 153, 128

Cuntz, M., \& Guinan, E. F. 2016, ApJ, 827, 79

del Burgo, C., \& Allende Prieto, C. 2016, MNRAS, 463, 1400

Dittmann, J. A., Irwin, J. M., Charbonneau, D., \& Berta-Thompson, Z. K. 2014, ApJ, 784, 156

Domagal-Goldman, S. D., Segura, A., Claire, M. W., Robinson, T. D., \& Meadows, V. S. 2014, ApJ, 792, 90

Dotter, A., Conroy, C., Cargile, P., \& Asplund, M. 2017, ApJ, 840, 99

Dressing, C. D., \& Charbonneau, D. 2015, ApJ, 807, 45

Dupuy, T. J., Kratter, K. M., Kraus, A. L., et al. 2016, ApJ, 817, 80

Eiroa, C., Marshall, J. P., Mora, A., et al. 2013, A\&A, 555, A11

Finch, C. T., \& Zacharias, N. 2016, AJ, 151, 160

Fontenla, J. M., Linsky, J. L., Witbrod, J., et al. 2016, ApJ, 830, 154

Foreman-Mackey, D., Hogg, D. W., Lang, D., \& Goodman, J. 2013, PASP, 125,306

France, K., Froning, C. S., Linsky, J. L., et al. 2013, ApJ, 763, 149

France, K., Linsky, J. L., Tian, F., Froning, C. S., \& Roberge, A. 2012, ApJL, 750, L32

France, K., Loyd, R. O. P., Youngblood, A., et al. 2016, ApJ, 820, 89

Gaia Collaboration, Brown, A. G. A., Vallenari, A., et al. 2018, A\&A, 616, A1

Gaia Collaboration, Prusti, T., de Bruijne, J. H. J., et al. 2016, A\&A, 595, A1 Gaidos, E., Mann, A. W., Lépine, S., et al. 2014, MNRAS, 443, 2561

Gray, R. O., Corbally, C. J., Garrison, R. F., et al. 2003, AJ, 126, 2048

Gray, R. O., Corbally, C. J., Garrison, R. F., et al. 2006, AJ, 132, 161

Gray, R. O., Napier, M. G., \& Winkler, L. I. 2001, AJ, 121, 2148

Guinan, E. F., Engle, S. G., \& Durbin, A. 2016, ApJ, 821, 81

Hardegree-Ullman, K. K., Cushing, M. C., Muirhead, P. S., et al. 2019, AJ, 158,75

Harman, C. E., Schwieterman, E. W., Schottelkotte, J. C., \& Kasting, J. F. 2015, ApJ, 812, 137

Hawley, S. L., Gizis, J. E., \& Reid, I. N. 1996, AJ, 112, 2799

Hébrard, G., \& Moos, H. W. 2003, ApJ, 599, 297

Heller, R., \& Armstrong, J. 2014, AsBio, 14, 50

Hu, R., Seager, S., \& Bains, W. 2012, ApJ, 761, 166

Huber, D., Bryson, S. T., Haas, M. R., et al. 2016, ApJS, 224, 2

Hussain, G. A. J., Allende Prieto, C., Saar, S. H., et al. 2006, MNRAS, 367, 1699

Jones, D. O., \& West, A. A. 2016, ApJ, 817, 1

Joyce, M., \& Chaboyer, B. 2018, ApJ, 864, 99

Kasting, J. F., Whitmire, D. P., \& Reynolds, R. T. 1993, Icar, 101, 108

Kastner, J. H., Sacco, G., Rodriguez, D., et al. 2017, ApJ, 841, 73

Kervella, P., Mérand, A., Pichon, B., et al. 2008, A\&A, 488, 667

Kopparapu, R. K., Ramirez, R., Kasting, J. F., et al. 2013a, ApJ, 765, 131

Kopparapu, R. K., Ramirez, R., Kasting, J. F., et al. 2013b, ApJ, 770, 82

Lalitha, S., Poppenhaeger, K., Singh, K. P., Czesla, S., \& Schmitt, J. H. M. M. 2014, ApJL, 790, L11

Lichtenegger, H. I. M., Lammer, H., Grießmeier, J.-M., et al. 2010, Icar, 210, 1

Lindegren, L., Hernández, J., Bombrun, A., et al. 2018, A\&A, 616, A2

Linsky, J. L., Draine, B. T., Moos, H. W., et al. 2006, ApJ, 647, 1106

Linsky, J. L., France, K., \& Ayres, T. 2013, ApJ, 766, 69

Loyd, R. O. P., \& France, K. 2014, ApJS, 211, 9

Loyd, R. O. P., France, K., Youngblood, A., et al. 2016, ApJ, 824, 102

Luck, R. E. 2017, AJ, 153, 21
Luger, R., \& Barnes, R. 2015, AsBio, 15, 119

Lurie, J. C., Henry, T. J., Jao, W.-C., et al. 2014, AJ, 148, 91

Mann, A. W., Feiden, G. A., Gaidos, E., et al. 2015, ApJ, 804, 64

Meshkat, T., Mawet, D., Bryan, M. L., et al. 2017, AJ, 154, 245

Miles, B. E., \& Shkolnik, E. L. 2017, AJ, 154, 67

Muirhead, P. S., Dressing, C. D., Mann, A. W., et al. 2018, AJ, 155, 180

Mulders, G. D., Pascucci, I., \& Apai, D. 2015, ApJ, 798, 112

Newton, E. R., Charbonneau, D., Irwin, J., et al. 2014, AJ, 147, 20

Newton, E. R., Charbonneau, D., Irwin, J., et al. 2015, ApJ, 800, 85

Pace, G. 2013, A\&A, 551, L8

Peacock, S., Barman, T., Shkolnik, E. L., et al. 2019, ApJ, 871, 235

Pecaut, M. J., \& Mamajek, E. E. 2013, ApJS, 208, 9

Pepe, F., Lovis, C., Ségransan, D., et al. 2011, A\&A, 534, A58

Perryman, M. A. C., Brown, A. G. A., Lebreton, Y., et al. 1998, A\&A, 331, 81

Plavchan, P., Werner, M. W., Chen, C. H., et al. 2009, ApJ, 698, 1068

Ramírez, I., Allende Prieto, C., \& Lambert, D. L. 2013, ApJ, 764, 78

Ramírez, I., Fish, J. R., Lambert, D. L., et al. 2012, ApJ, 756, 46

Rauer, H., Catala, C., Aerts, C., et al. 2014, ExA, 38, 249

Redfield, S., \& Linsky, J. L. 2008, ApJ, 673, 283

Reid, I. N., Cruz, K. L., Laurie, S. P., et al. 2003, AJ, 125, 354

Reid, I. N., Hawley, S. L., \& Gizis, J. E. 1995, AJ, 110, 1838

Rice, J. B., \& Strassmeier, K. G. 1998, A\&A, 336, 972

Richey-Yowell, T., Shkolnik, E. L., Schneider, A. C., et al. 2019, ApJ, 872, 17

Ricker, G. R., Winn, J. N., Vanderspek, R., et al. 2015, JATIS, 1, 014003

Rojas-Ayala, B., Covey, K. R., Muirhead, P. S., et al. 2012, ApJ, 748, 93

Ross, F. E. 1929, AJ, 39, 140

Ross, F. E. 1939, AJ, 48, 163

Rugheimer, S., Kaltenegger, L., Segura, A., Linsky, J., \& Mohanty, S. 2015, ApJ, 809, 57

Saur, J., Fischer, C., Wennmacher, A., et al. 2018, ApJ, 859, 74

Schneider, A. C., \& Shkolnik, E. L. 2018, AJ, 155, 122

Schuster, W. J., Moitinho, A., Márquez, A., Parrao, L., \& Covarrubias, E. 2006, A\&A, 445, 939

Segura, A., Walkowicz, L. M., Meadows, V., Kasting, J., \& Hawley, S. 2010, AsBio, 10, 751

Shkolnik, E., Liu, M. C., \& Reid, I. N. 2009, ApJ, 699, 649

Shkolnik, E. L., Anglada-Escudé, G., Liu, M. C., et al. 2012, ApJ, 758, 56

Shkolnik, E. L., Rolph, K. A., Peacock, S., \& Barman, T. S. 2014, ApJL, 796, L20

Silva Aguirre, V., Davies, G. R., Basu, S., et al. 2015, MNRAS, 452, 2127

Sing, D. K., Vidal-Madjar, A., Désert, J.-M., et al. 2008, ApJ, 686, 658

Skrutskie, M. F., Cutri, R. M., Stiening, R., et al. 2006, AJ, 131, 1163

Stassun, K. G., Oelkers, R. J., Pepper, J., et al. 2018, AJ, 156, 102

Tian, F., France, K., Linsky, J. L., Mauas, P. J. D., \& Vieytes, M. C. 2014, E\&PSL, 385, 22

Torres, C. A. O., Quast, G. R., da Silva, L., et al. 2006, A\&A, 460, 695

Tuomi, M., Jones, H. R. A., Anglada-Escudé, G., et al. 2019, arXiv:1906. 04644

Valenti, J. A., \& Fischer, D. A. 2005, ApJS, 159, 141

van Altena, W. F., Lee, J. T., \& Hoffleit, E. D. 1995, The General Catalogue of Trigonometric Stellar Parallaxes (New Haven, CT: Yale Univ. Press)

van Leeuwen, F. 2007, A\&A, 474, 653

Waalkes, W. C., Berta-Thompson, Z., Bourrier, V., et al. 2019, AJ, 158, 50

Wood, B. E., Redfield, S., Linsky, J. L., Müller, H.-R., \& Zank, G. P. 2005 , ApJS, 159, 118

Yee, S. W., Petigura, E. A., \& von Braun, K. 2017, ApJ, 836, 77

Youngblood, A., France, K., Loyd, R. O. P., et al. 2016, ApJ, 824, 101

Youngblood, A., France, K., Loyd, R. O. P., et al. 2017, ApJ, 843, 31

Zuckerman, B., \& Song, I. 2004, ARA\&A, 42, 685 